\title{
Primary Headache in Children Aged Between 6 and 18 Years-Causes and Associated Factors
}

\author{
Abdul Rahman Abul Hassan ${ }^{1}$. Anita Palani ${ }^{1}$ - Saji James ${ }^{1}$. Ranjith Kumar Manokaran ${ }^{2}$. \\ Thasma Santhanakrishnan Arunprasath ${ }^{1}$ []
}

Received: 2 October 2021 / Accepted: 24 December 2021/Published online: 3 March 2022

(c) Dr. K C Chaudhuri Foundation 2022

To the Editor: Headache is a common problem in children and adolescents and is a major cause of school absenteeism and impairment in quality of life [1]. Lack of appropriate management of primary headache in children exists [2]. Knowledge about prevalence of primary headache and its subtypes is essential in executing appropriate level of care. This study was done in a tertiary care university hospital to describe the causes and associated factors of primary headache in children aged between 6 and $18 \mathrm{y}$.

Children in the age group of 6 to $18 \mathrm{y}$, with diagnosis of primary headache as per the International Classification of Headache Disorders (ICHD), were included in the study. Clinical details, headache characteristics, and associated factors were noted. Pain severity was assessed using Wong-Baker "faces" rating scale. Characteristics and associated factors were compared between the subtypes of primary headache.

A total of 251 children (males, $n=111,44.2 \%$ ) with primary headache presented during the study period; 136 (54.2\%) children had migraine and 115 (45.8\%) children had tension-type headache (TTH). Children with migraine in comparison with TTH were younger in age $(10.52 \pm 2.84$ vs. $14.50 \pm 2.12, p<0.001)$. This finding is in contrast with previous studies [3]. This difference may be due to different psychosocial environment of the study population. Children with migraine in comparison with TTH had increased need of drug for headache relief ( $100 \%$ vs. $71.3 \%, p<0.001)$ and increased frequency of self-medication $(72.8 \%$ vs. $54.8 \%$,

Thasma Santhanakrishnan Arunprasath drtsarunprasath@yahoo.com

1 Department of Paediatrics, Sri Ramachandra Institute of Higher Education and Research, Porur, Chennai, Tamil Nadu 600116, India

2 Division of Pediatric Neurology, Department of Neurology, Sri Ramachandra Institute of Higher Education and Research, Porur, Chennai, Tamil Nadu, India $p<0.001)$. This finding has been reported previously [4]. This may be due to monthly episodes of migraine, which are prolonged and severe, which is observed in our study also. Identification of these children and instituting appropriate care is important in terms of preventing morbidity.

This was a hospital based study, which may not truly reflect the status in general population. Large scale studies need to be done at population level.

\section{Declarations}

Conflict of Interest None.

\section{References}

1. Nodari E, Battistella PA, Naccarella C, Vidi M. Quality of life in young Italian patients with primary headache. Headache. 2002;42:268-74.

2. Shuaibi S, AlAshqar A, Ahmed SF, et al. Primary headache disorder among school students in Kuwait. Front Neurol. 2021:12:621017.

3. Genizi J, Bugdnoskya V, Aboud A, et al. Migraine and tensiontype headache among children and adolescents: application of International Headache Society Criteria in a clinical setting. J Child Neurol. 2021;36:618-24.

4. Brusa P, Allais G, Scarinzi C, et al. Self-medication for migraine: A nationwide cross-sectional study in Italy. PLoS One. 2019;14:e0211191.

Publisher's Note Springer Nature remains neutral with regard to jurisdictional claims in published maps and institutional affiliations. 